\title{
Les préfaces du Dictionaire Francoislatin de Robert Estienne (1539 et 1549)
}

La place de la langue vernaculaire à l'aube de la lexicographie française.

\section{Sabine Lardon}

\section{(2) OpenEdition}

\section{Journals}

Édition électronique

URL : https://journals.openedition.org/eve/680

DOI : $10.4000 /$ eve.680

ISSN : 2425-1593

Éditeur :

Université de Savoie, Université Jean Moulin - Lyon 3

\section{Référence électronique}

Sabine Lardon, « Les préfaces du Dictionaire Francoislatin de Robert Estienne (1539 et 1549) », Corpus

Eve [En ligne], Domaine français, mis en ligne le 18 octobre 2013, consulté le 16 avril 2022. URL :

http://journals.openedition.org/eve/680 ; DOI : https://doi.org/10.4000/eve.680

Ce document a été généré automatiquement le 16 avril 2022.

(c) Tous droits réservés 


\section{Les préfaces du Dictionaire Francoislatin de Robert Estienne (1539 et 1549)}

La place de la langue vernaculaire à l'aube de la lexicographie française.

\section{Sabine Lardon}

\section{RÉFÉRENCE}

Robert Estienne, Dictionaire Francoislatin, contenant les motz \& manieres de parler Francois, tournez en Latin. À Paris, De l'imprimerie de Robert Estienne, 1539, [préface sans titre]. Robert Estienne, Dictionaire Francoislatin, autrement dict Les mots Francois, avec les manieres d'user d'iceulx, tournez en Latin. Corrigé \& augmenté. À Paris, De l'imprimerie de Robert Estienne Imprimeur du Roy, 1549, « Au lecteur ».

\section{Notice biographique}

1 Robert Estienne est né en $1503^{1}$, probablement à Paris (« au cœur du Quartier Latin » selon P. Aquilon ${ }^{2}$ ). Il est le fils de Henri Estienne (père), premier imprimeur du nom, dont les enfants, François, Robert et Charles, embrassèrent tous la profession. Après avoir collaboré avec Wolfgang Hopyl, Henri Estienne père fonde son imprimerie dans les années 1503-1504, au moment de la naissance de son fils Estienne. Il a pour amis et protecteurs Guillaume Budé, François et Guillaume Briçonnet, l'évêque Jean du Bellay, Jean Ganay et surtout Jacques Lefèvre d'Étaples. À défaut de témoignages précis sur l'éducation reçue par Robert Estienne, ces noms sont riches d'enseignement et permettent de mesurer le milieu culturel, humaniste et évangélique, dans lequel il a grandi. Henri Estienne père est mort en 1520. Dès 1521, sa veuve épouse Simon de Colines, qui reprend l'imprimerie. Formé aux côtés de son père, puis de son beau-père, Robert Estienne se voit confier les révisions du Nouveau Testament en latin, publié en 
1523. En 1526, il reprend seul l'entreprise familiale, tandis que Simon de Colines s'établit à son propre compte. En 1528 (1526 selon P. Aquilon ${ }^{3}$ ), il épouse Perrette Bade, une jeune femme cultivée, fille de l'imprimeur Josse Bade, doyen des libraires universitaires de Paris, qui lui donne neuf enfants ${ }^{4}$. Perpétuellement en butte aux poursuites de la Sorbonne en raison de son travail d'édition des textes sacrés (voir cidessous), Robert Estienne finit par se réfugier à Genève dans les années 1550-1551 (son mariage, en secondes noces, avec Marguerite Duchemin est enregistré sur les registres de la ville le 14 décembre 15505). Il y imprime les œuvres de Calvin et de Théodore de Bèze avec lesquels il se lie. Son activité lui vaut d'être admis gratuitement comme citoyen par la ville de Genève, en 1556. Il meurt le 7 septembre 1559, à l'âge de 56 ans. Son fils aîné Henri reprendra l'entreprise familiale et poursuivra l'œuvre de son père, tandis que ses deux autres fils, Robert et Charles, restés catholiques ${ }^{6}$, se voient déshérités.

Robert Estienne a consacré l'essentiel de son activité éditoriale à trois tâches principales. Il est un humaniste, voué à la cause des belles lettres et des auteurs classiques. La notice biographique que lui consacre La Croix du Maine dans sa Bibliothèque $e^{7}$ le souligne, en rappelant sa connaissance des langues hébraïque, grecque et latine ainsi que des auteurs antiques:

Robert Estienne Parisien, pere de Henry Estienne, tous deux imprimeurs très-doctes et des plus renommez de toute l'Europe, pour le profit qu'ils ont fait en la correction et impression des plus excellents autheurs, Hebreux, Grecs et Latins, etc. Cettui-cuy nommé Robert estoit for[t] docte ès langues Hebraïque, Grecque et Latine, outre celà en la Françoise, en laquelle il a composé un traicté de grammaire, imprimé par luy-mesmes [sic], et depuis par son autre fils, nommé François l'an 1569.

$[\ldots]$

Il mourut à Genesve l'an 1559. le septiesme jour de Septembre, âgé de 56. ans. Je feray mention de ses escrits Latins autre-part.

Dès les premières années, Robert Estienne édite ainsi Cicéron (Epistolarum familiares libri XVI, en 1526 ; Oratorice Partitiones \& de optimo genere Oratorum en 1527 etc.) ; la vie et les fables d'Ésope en 1527; les comédies de Térence en 1529, accompagnées du commentaire de Donat ; celles de Plaute en 1530 ; les œuvres complètes de Virgile avec le commentaire de Servius en $1532^{8}$, puis celles de Cicéron (M.T.Ciceronis Opera), réunies en quatre volumes in-folio en 1538-1539, et dont il offre ensuite une version portative en neuf volumes in-8 en 1543-1544. Ces travaux nourriront les éditions successives de son Thesaurus Linguce latince.

Robert Estienne a également mis sa connaissance et sa pratique des langues hébraïque, grecque et latine au service d'une diffusion des textes sacrés. Dès 1528 , il témoigne de ses convictions évangéliques en publiant un texte du réformateur suisse Zwingli (Quo pacto ingenui adolescentes formandi sint prceceptiones). Ainsi que le rappelle Martine Furno", «toute sa vie tant personnelle qu'intellectuelle ne sera qu'une marche vers l'épanouissement et l'affirmation de cette foi ». Il édite ainsi la Bible en latin, dans la version de la Vulgate en 1528; les textes hébraïques (Biblia Hebraica) de 1539 à 1542 (version in-folio, suivie d'une édition portative en huit volumes in-16 en 1546); le Nouveau Testament en grec (Novum testamentum grcecum) en 1546 (rééd. en 1549). À l'érudition, Robert Estienne associe un souci pédagogique permanent. En réponse au désir des premiers réformateurs français d'amener chacun à méditer les Écritures, il donne, dès 1534, une édition de la Bible en format portatif (in-8). Il édite en placards 
(affiches) in-folio destinés aux écoles les Dix Commandements et une Summa totius Scriptur $\mathfrak{e}^{10}$ en latin ainsi qu'en français. Il apporte aux textes sacrés l'éclairage des professeurs de l'époque, ajoutant en marge de l'édition de la Bible latine de 1545 les annotations sur l'Ancien Testament de Vatable, professeur au Collège Royal fondé par François $I^{\text {er }}$ en $1540^{11}$. Cette publication des textes sacrés lui valut d'incessantes poursuites de la part de la Sorbonne, qui le contraignirent à se réfugier à Genève dans les années 1550-1551 et auxquelles il répondit par une "Réponse aux censures des Théologiens de Paris $»^{12}$. À Genève, il poursuit et achève son travail, éditant les grandes bibles calvinistes de Calvin et de Bèze.

Robert Estienne a également montré un intérêt constant pour les ouvrages consacrés à la langue (alphabet, grammaire, dialectique, rhétorique, dictionnaire,...) et à son enseignement, en publiant de nombreux ouvrages pédagogiques visant tantôt de jeunes apprenants, tantôt des lecteurs plus avancés. Sur les neuf ouvrages parus la première année, en 1526, l'on relève ainsi deux éditions du Libellus de accentibus de Priscien, la Grammatica latina de Mélanchton, un Ars versificatoria de Hulrich von Hutten et le De octo partibus orationis de Donat. Robert Estienne lui-même rédigea plusieurs ouvrages destinés à l'étude de la langue française : La maniere de tourner en Langue Francoise les Verbes actifz, passifz, gerundifz, Supins et Participes (éd. princeps 1528), De Gallica verborum declinatione (1540), La maniere de tourner touts especes de noms latins, en nostre langue francoyse (1537), Les Declinaisons des noms et verbes (1546), Les mots francois selon l'ordre des lettres (1543) et le Traicté de la grammaire Francoise (1557).

6 Son activité de typographe (et en particulier ses travaux d'édition de la Bible en latin et en grec) lui valut d'être nommé par François $\mathrm{I}^{\mathrm{er}}$ «Imprimeur \& libraire ès lettres hebraiques \& Latines » en juin $1539^{13}$. Si ce dernier titre existait déjà, le premier, relatif aux lettres hébraïques, fut créé pour lui ${ }^{14}$. Il succède également à Conrad Néobar, mort en 1540, au titre d'imprimeur pour les lettres grecques. Si, en l'absence de traces officielles, il est difficile de dater précisément son accession à ce titre, E. Armstrong la repousse néanmoins au printemps 1542 au plus tôt, R. Estienne continuant d'employer jusque-là la mention "Rob. Stephanus hebraicarum et latinarum literarum typographus regius ${ }^{15}$, alors qu'il n'emploiera plus par la suite que la mention englobante de "typographus regius", qui fait toutefois sa première apparition dans la Bible de 1540. François $\mathrm{I}^{\text {er }}$ (à l'instigation de l'évêque Pierre Duchâtel qui succède à Budé comme garde de la librairie du roi, selon P. Aquilon ${ }^{16}$ ), fait graver pour son imprimeur des fontes de caractères grecs par Claude Garamond, afin de permettre l'édition des manuscrits grecs de Fontainebleau. Le premier texte imprimé avec les "grecs du Roi » paraît en 1544, contenant l'édition grecque princeps de l'Histoire ecclésiastique d'Eusèbe de Césarée (jusqu'alors imprimée dans des traductions latines) et six autres œuvres historiques ${ }^{17}$.

\section{Présentation du contexte}

\section{Robert Estienne lexicographe et les débuts de la lexicographie française}

7 Les premiers ouvrages lexicographiques étaient en latin (les deux principales manifestations en sont l'Elementarium doctrince erudimentum de Papias Vocabulista et les Magnce derivationes de Uguccione da Pisa [Huguccio de Pise]) et constituaient des compilations encyclopédiques des connaissances de l'époque. Le premier à y avoir 
introduit un classement alphabétique fut un moine dominicain italien, Giovanni de Balbi (Jean de Gênes), dans sa somme scientifique: Summa quae vocatur Catholicon, achevée en 1286. Il faut ensuite attendre la fin du XIII e siècle pour trouver un lexique alphabétique latin-français. Mario Roques, qui a constitué un recueil des lexiques médiévaux (XII ${ }^{-}-\mathrm{XV}^{\mathrm{e}}$ siècles), l'a intitulé Abauus d'après le premier mot qui y figure ${ }^{18}$. À la fin du $\mathrm{XV}^{\mathrm{e}}$ siècle, figurent parmi les incunables, les lexiques qui avaient été les plus diffusés sous forme manuscrite. Le premier dictionnaire imprimé fut ainsi le Catholicon, édité à Mayence dès 1460. S'y ajoutent des approches nouvelles, comme les commentaires lexicaux de Perotti, Cornucopice sive linguce latince (achevés avant 1480, publication posthume en $1489^{19}$ ), qui induisent une approche critique et sélective du latin. Concernant le début du XVI ${ }^{\mathrm{e}}$ siècle, Margarete Lindermann ${ }^{20}$ signale l'importance, trop souvent négligée, du dictionnaire latin-espagnol d'Antonio Nebrija (1492) qui « est à la base d'un dictionnaire latin-français qui fut édité pour la première fois à Lyon par Simon Vincent en 1511»: le Vocabularius nebrissensis lequel connut ensuite de nombreuses rééditions. Contrairement à ce que son titre pourrait laisser paraître, cet ouvrage, s'il reprend, selon les observations de M. Lindermann, la nomenclature latine $\mathrm{du}$ dictionnaire latin-espagnol de Nebrija, est un dictionnaire latin-français, plus conséquent que les premiers dictionnaires correspondants de Robert Estienne.

L'activité de lexicographe de Robert Estienne débute vers 1527 ou 1528, quand il se voit demander de rééditer le Dictionarium d'Ambrogio da Calepio (Calepin). Cet ouvrage, paru à Reggio en 1502, s'était vite imposé auprès d'un public étudiant, mais ses multiples rééditions (qui se prolongent tout au long du siècle) avaient contribué à l'altérer. C'est pourquoi R. Estienne explique, dans la préface de son Thesaurus lingce latince (1531), que plutôt que de le corriger, il conçut l'idée de cet ouvrage nouveau ${ }^{21}$. Une deuxième édition augmentée paraît en 1536, puis une troisième, portée à trois volumes in-folio, en 1543. À la fin de sa vie, Robert Estienne conçut le projet de réaliser un travail identique pour la langue grecque, mais il ne put le mener à bien et c'est son fils, Henri, qui acheva l'entreprise ${ }^{22}$. Entre ces deux termes, l'activité de lexicographe de Robert Estienne s'est déclinée selon les étapes suivantes :

1531 : Dictionarium seu Latince linguce Thesaurus. Réédité et augmenté en 1536 et 1543.

1538 : Dictionarium latinogallicum. Réédité en 1546, 1552 et 1570.

1539 : Dictionaire francoislatin. Réédité en 1549.

1542 : Dictionariolum puerorum (latin-français). Réédité en 1557.

1544 : Les Mots francois selon l'ordre des lettres, ainsi que les fault escrire tournez en latin pour les enfants. Réédité en 1557 sous le titre Petit dictionaire des mots francois et en 1559 sous le titre Petit dictionnaire françois latin, autrement dict les mots françois.

Si le Thesaurus constitue le grand œuvre lexicographique de Robert Estienne, c'est toutefois sur son Dictionaire Francoislatin (1539) que s'attarde François Grugé de La Croix du Maine, lequel consacre deux paragraphes, en 1584, au succès rencontré par l'ouvrage.

Cettui-cuy nommé Robert estoit for[t] docte és langues Hebraïque, Grecque et Latine, outre celà en la Françoise, en laquelle il a composé un traicté de grammaire, imprimé par luy-mesmes [sic], et depuis par son autre fils, nommé François l'an 1569.

Il a davantage composé le dictionnaire François-Latin, auquel les mots François, avecques les manieres d'user d'iceux, sont tournés en Latin.

Ce livre a esté imprimé par plusieurs fois, et depuis imprimé avec les additions de Jean Thierry de Beauvais, Jean le Frere de Laval, et Jean Nicot Conseiller du Roy, lequel l'a beaucoup augmenté en la derniere edition, imprimée chez Jaques du Puis à Paris, et autres ${ }^{23}$. 
Ce glissement dans le regard porté sur l'œuvre lexicographique d'Estienne tient à la nature même du projet du bibliothécaire, explicité par le titre de son recensement bibliographique : Premier volume de La Bibliotheque du sieur de la Croix du Maine. Qui est un catalogue general de toutes sortes d'Autheurs, qui ont escrit en François depuis cinq cents ans et plus, jusques à ce jourd'huy. L'organisation de sa notice tend donc à montrer que l'intérêt porté par l'imprimeur du roi aux langues «Hebraïque, Grecque et Latine » ne lui a pas fait négliger la sienne, à laquelle il a consacré une grammaire et un dictionnaire. Il y a là une reconstruction de l'œuvre stéphanienne délibérément revue à la lueur du projet de défense et d'illustration de la langue française entrepris par La Croix du Maine, qui parvient ainsi à hausser le français à l'égal des langues anciennes dans les préoccupations de l'imprimeur royal.

\section{La place du français en question dans le Dictionaire Francoislatin de Robert Estienne}

Ce statut privilégié du Dictionaire Francoislatin est réaffirmé un an plus tard (en 1585) par le second des grands bibliothécaires de cette fin de siècle, Antoine du Verdier. Son objectif est le même que celui de son confrère : dresser le Catalogue de tous ceux qui ont escrit, ou traduict en François, \& autres Dialectes de ce Royaume et son témoignage fait, avant toute chose, du Dictionaire Francoislatin un inventaire de la langue française :

Dictionaire Françoïs Latin, contenant les mots et manieres de parler François, tournez en Latin. Il en a esté fait de plusieurs sortes, et imprimé en divers lieux.

Robert Estienne a fait le premier ${ }^{24}$.

L'on peut hésiter entre plusieurs lectures de sa notice (l'on pourrait ainsi comprendre que R. Estienne a réalisé l'édition princeps d'une entreprise qui a été poursuivie par d'autres, allusion possible aux continuations posthumes). L'une d'elle investit R. Estienne en père de la lexicographie française, dont il aurait réalisé le premier dictionnaire, ensuite imité. L'ouvrage est le premier, de fait, à porter le titre français de «Dictionaire » (voué à une longue destinée, mais avec un seul « $\mathrm{n}$ » pour l'heure) et à présenter des entrées alphabétiques en langue vernaculaire ${ }^{25}$. Cette place, aux origines de la lexicographie française, lui est encore reconnue de nos jours:

Le Dictionnaire français-latin de 1539 est le pendant de la version latin-français antérieure. Grâce aux enrichissements du contenu français introduits dans cette dernière, il a pu présenter le premier inventaire étendu du vocabulaire de la langue vulgaire. À ce titre, il prend place à la tête de la lexicographie française tout entière qui en procède directement ou indirectement ${ }^{26}$.

Elle nécessite toutefois certaines nuances, dans la mesure où ce dictionnaire, ainsi que le rappelle Jean Pruvost, est encore bilingue et qu'il faudra attendre le XVII ${ }^{\mathrm{e}}$ siècle pour que l'évolution aboutissant au dictionnaire monolingue arrive à son terme ${ }^{27}$.

11 Martine Furno a montré, au fil de plusieurs articles, que le Dictionaire Francoislatin d'Estienne, plus encore qu'un point de départ, est bien plutôt un point d'aboutissement ${ }^{28}$ : celui d'une entreprise lexicographique avant tout latine qui débute avec le Thesaurus Linguce latince en 1531. Dans cet ouvrage fondateur, destiné à des latinistes avancés, le français n'occupe qu'une part réduite, n'intervenant que ponctuellement, là où la définition exclusivement latine pourrait porter à confusion, pour des termes techniques par exemple (comme des noms de plante ou d'arbre) ${ }^{29}$. Pour un public de niveau intermédiaire, R. Estienne va alors concevoir une version simplifiée, le Dictionarium latino-gallicum de 1538, «qui ne conserve qu'une partie des exemples latins d'origine » et "les double quasi systématiquement d'une traduction 
française qui en éclaire les points difficiles $\star^{30}$. Un an plus tard, le Dictionaire Francoislatin (1539) n'en constitue pas seulement le pendant, mais plus encore le retournement, par rétroversion systématique des entrées comme des citations (traduction française des auteurs latins cités dans le Dictionarium latino-gallicum). Ces deux dictionnaires connaitront donc parallèlement d'une part une réédition augmentée (1546 pour le Dictionarium latino-gallicum, 1549 pour le Dictionaire Francoislatin), d'autre part et à l'inverse une réduction simplificatrice (le Dictionariolum puerorum en 1542 et Les Mots françois selon l'ordre des lettres en 1544), ce qui permet à R. Estienne de décliner son entreprise lexicographique sur trois niveaux: pour un public débutant, intermédiaire puis avancé, selon une double ramification (latine > française et française > latine), dont le Thesaurus Linguce Latince $(1531,1536,1543)$ constitue le tronc commun (point de départ de l'entreprise lexicographique, en même temps que point d'aboutissement destiné au lecteur le plus avancé) :

- Dictionarium latino-gallicum (édition princeps 1538, édition augmentée 1546); Dictionariolum puerorum (1542).

- Dictionaire Francoislatin (édition princeps 1539, édition augmentée 1549); Les Mots françois selon l'ordre des lettres (1544).

Tous ces dictionnaires sont donc liés dans cette chaîne dont le Dictionaire Francoislatin constitue un maillon concourant au même but que les autres : permettre la maîtrise du latin.

Il est clair que pour Robert Estienne ces ouvrages sont des moyens, non des fins : ils permettent de donner accès à une première connaissance du latin, qui, peu à peu perfectionnée, pourra une fois maitrisée donner la pleine mesure de son activité dans une écriture et une compréhension directe des textes, accompagnée par la somme doctorale du Thesaurus linguce latine ${ }^{31}$.

Les deux ouvrages, le Dictionarium et le Dictionaire, sont en conséquence destinés à être utilisés conjointement et de manière complémentaire par le latiniste de niveau intermédiaire : «l'un pour éclairer sa lecture, l’autre pour secourir rapidement son écriture. ${ }^{32}$ »

13 Le choix du titre même semble le confirmer. Ce nom, destiné à s'imposer dans la lexicographie française, fait là sa première apparition, comme simple décalque encore du dictionarium latin ${ }^{33}$. Martine Furno ${ }^{34}$, qui étudie les différents titres donnés aux ouvrages lexicographiques: thesaurus, lexicon, vocabularium, dictionarium, dictionnaire, thrésor..., le rappelle, pour constater également qu'il s'agit d'un terme non marqué, à la différence du thesaurus, dont le titre délibérément métaphorique (et emprunté par Estienne le premier à d'autres domaines que la lexicographie), est choisi pour connoter la richesse du contenu, et donc de la langue latine (Jean Pruvost rappelle toutefois que le terme «diction(n)aire » s'oppose également à «lexicon» et à "vocabularium » qui seront respectivement francisés en « lexique » et «vocabulaire » et qui désignent des ensembles de moindre importance ${ }^{35}$ ). C'est pourquoi d'ailleurs, le Thesaurus fonde son recensement lexical sur un dépouillement systématique des auteurs latins au fur et à mesure des éditions que leur consacre R. Estienne. Les préfaces des trois éditions successives du Thesaurus mettent ce travail en valeur: dans celle de 1531, Estienne explique le sytème de référence pour Térence (acte, scène, vers), Plaute (scène, vers) et Pline le Jeune (numéro de la lettre); en 1536, il y ajoute Cicéron (lettres et discours), Caton, Varron, Columelle, Pline l'Ancien, César, Tite Live et Martial; en 1543, Perse, Juvénal, Virgile et «reliquisque poetis» (les autres poètes) ${ }^{36}$. Conçu comme un «conservatoire linguistique», le Thesaurus n'est pas pour autant un "conservatoire 
immobile ", tombeau élevé à la mémoire d'une langue morte : à cette date, sa fin est encore « la pratique active de la langue », le latin étant toujours parlé et surtout écrit ${ }^{37}$.

Les enrichissements de 1549, s'ils donnent au Dictionnaire françois-latin un peu plus de personnalité propre, ne changeront rien au principe de départ: le but primordial de ce dictionnaire est bien d'écrire du latin, non d'expliquer du français. Même si la langue d'entrée, le français, est traitée avec attention, l'objet des soins de l'auteur est la langue de sortie, le latin. On ne trouve donc pas dans le Dictionnaire françois-latin, d'extraits d'auteurs français pour illustrer l'usage vernaculaire, mais des tournures françaises forgées à partir des auteurs latins, et rendues ensuite dans leur langue d'origine comme autant de modèles. Ce statut du Dictionnaire françoislatin, élément d'une œuvre lexicographique latine, ne doit jamais être oublié lorsqu'on en étudie des extraits ${ }^{38}$.

Nous sommes loin encore d'un projet d'illustration de la langue nationale et l'on comprend mieux dès lors cette déclaration de Charles Beaulieux: "Le thresor de la langue francoise [1606], de Nicot, est véritablement le premier dictionnaire français. Avant Nicot, personne n'avait éprouvé le besoin de réunir en un corps les richesses de notre langue trop longtemps dédaignée. ${ }^{39}$ " Du Thesaurus linguce latince d'Estienne au Thresor de la langue francoise de Nicot cependant, le chemin passe par le Dictionarium latino-gallicum et le Dictionaire Francoislatin. En étant le premier à opérer le retournement, R. Estienne a ouvert la voie, et même si son but premier n'était pas l'illustration du français, il a prouvé de facto que le retournement était possible et que la lexicographie française pouvait se poser en pendant d'un ouvrage de lexicographie latine.

Sa position à l'égard du français est certes ambiguë, voire peu flatteuse. C'est paradoxalement en effet dans le Dictionarium latino-gallicum qu'Estienne évoque la richesse de la langue française : "Quid enim aut ad meorum ciuium excitanda ex litteris studia maius,...quam...latentem adhuc linguæ nostræ gazam exponere ? ${ }^{40} »$ Martine Furno attire toutefois l'attention sur le fait qu'Estienne emploie ici en latin le mot grec et rare de « gaza » plutôt que « thesaurus » qu'il réserve à son dictionnaire monolingue latin (ou à bilinguisme réduit).

16 Il n'en reste pas moins que Robert Estienne semble s'être incliné devant la nécessité d'en passer désormais par la langue nationale, celle qu'il appellera "nostre langue Francoise » dans la préface de son Traicté de la grammaire Francoise de 1557. Et cela, dès le stade même du Thesaurus, le grand œuvre latin, pourtant destiné à un public avancé :

Eo uere in opere, sermone uti Gallico (quamquam ne id quidem ualde ab instituto nostro abhorrere uidebatur), nostri certe consilii non erat, nisi certorum hominum a quibus dissentire non poeteram quique magno id a me studio contenderent, uoluntati parendum omnino fuisset, cum prcesertim et honesta peterent et iis fortasse etiam non parum utilitatis allatura, qui cum uulgo bene docti uideri uelint, magna id libertate reprenhendunt. Tantum illa quce in promptuu et sine circonlocutione occurrerunt, non parum multa consulto prcetermittentes, adiecimus ${ }^{41}$.

R. Estienne reconnaît, non sans une certaine ambiguïté, avoir cédé à ceux qui le lui réclamaient bien que cela n'ait pas été dans son dessein premier, tout en reconnaissant que cela puisse être utile, y compris à ceux qui critiquent cette démarche. Le détour par le français dans l'apprentissage du latin a donc ses partisans comme ses détracteurs et l'on sent la position inconfortable de R. Estienne dans cette préface, partagé sans doute entre son dévouement aux lettres antiques et son pragmatisme didactique. Toujours est-il que ce bilinguisme d'abord restreint, dans le Thesaurus, puis systématisé et dédoublé dans le Dictionarium et le Dictionaire, semble attester que l'apprentissage et la 
pratique mêmes du latin ne vont peut-être plus désormais sans le passage, plus ou moins poussé (selon le niveau de l'apprenant), par la langue vernaculaire. C'est la conclusion à laquelle aboutit $\mathrm{M}$. Furno :

Mais, au-delà de cette description, ce qui fait l'intérêt des dictionnaires bilingues d'Estienne, et surtout de leur maturation depuis 1531, c'est ce qu'ils disent, plus empiriquement qu'explicitement, des idées stéphaniennes de l'apprentissage du latin. En effet, l'intention d'offrir, dès 1531, un ouvrage qui ne soit pas un rudiment, mais un dictionnaire "scientifique », et qui comporte aussi des éclaircissements en vernaculaire est bien la reconnaissance, de fait, que l'apprentissage et la pratique de la langue se font, désormais, avec le vernaculaire, et, d'une certaine façon, à travers lui : c'est en transposant le latin en français qu'on le comprend sûrement, et on le pense en français, si je puis dire, avant de l'écrire ${ }^{42}$.

Premier dictionnaire du français, mais destiné à l'usage de latinistes, le Dictionaire se pose à l'intersection d'une histoire lexicographique, à un moment où le latin est encore une langue pratiquée (à l'oral et plus encore à l'écrit), mais dont l'acquisition passe désormais de plus en plus par le français, amenant des prises de positions divergentes. Dans cette histoire, R. Estienne a tranché : bon gré mal gré, le grand éditeur humaniste, guidé par l'intérêt qu'il porte aux œuvres didactiques, reconnaît l'intérêt d'en passer désormais par le français, y compris (et même à un degré réduit) pour les apprenants avancés, témoignant par là du terrain gagné par le vernaculaire sur le véhiculaire. Son entreprise est indissociable d'un contexte: Edgar E. Brandon comme Jean Pruvost le rappellent ${ }^{43}, 1539$ est l'année de l'ordonnance de Villers-Cotterêts, promulguée par François $\mathrm{I}^{\mathrm{er}}$ et imposant le français comme langue administrative. Imprimeur du roi, Robert Estienne va donc progressivement s'impliquer dans cette entreprise linguistique.

\section{Enjeux linguistiques du texte}

\section{La préface de l'édition de 1539}

Le but principal du Dictionnaire Francoislatin est d'emblée précisé, à l'ouverture de la préface de 1539. À ce stade, point de désir d'illustration de la langue nationale : il s'agit de faire " quelque chose qui soit utile en-general à tous ceulx qui entendent au faict des lettres» [1]. La portée de l'entreprise est toutefois aussitôt nuancée (« ou pour le moins »), l'auteur visant, par-delà cet intérêt "général », un lectorat spécifique. Le propos se développe ici en chiasme, de manière à ramener à deux reprises l'ouvrage à l'enseignement du latin :

[1] ou pour le moins duisant aux apprentiz pour lesquelz il fault d'autant plus soigner, qu'ilz ont greigneur besoing de secours, que ceulx qui ont acquiz quelque ruze, et peuvent d'eulx mesmes, sans l'aide d'autruy, passer par les destroictz de la langue Latine : [2] À ceste cause après avoir mis en lumiere le grand Thresor d'icelle langue, lequel peult servir à toutes gens de quelque hault scavoir qu'ilz soyent garniz : nous avons mis cueur et entente au soulagement de la jeunesse Francoise, qui est sur son commencement et bachelage de literature.

L'ouvrage est donc en premier lieu destiné aux jeunes lecteurs en cours d'apprentissage du latin, qu'ils ne maîtrisent encore qu'imparfaitement ("apprentiz ", " commencement» et «bachelage»). Aux plus avancés et aux savants (les "gens de quelque hault scavoir »), R. Estienne dédie son Thesaurus. C'est bien ici la langue latine qui sert de pivot syntaxique et logique (« À ceste cause ») à la progression du propos et à la présentation de l'entreprise. Le Dictionnaire Francoislatin n'est donc pas seulement le 
retournement du Dictionarium latino-gallicum, mais bien son indissociable complément dans une entreprise commune, destinée à un même lectorat (rassemblé ci-dessous dans un «leur» collectif). La progression logique de la préface («si », au sens de «en conséquence ») et le parallélisme binaire de la construction le confirment :

[3] Si leur avons faict deux livres: L'ung commenceant par les motz Latins deschiffrez en Francois : qui fut publié dès l'année precedente. L'autre est cestuy cy qui va prenant les motz de la langue Francoise, les mettant après en Latin tout au plus près qu'il s'est peu faire.

Dans la suite de la préface toutefois, le discours se fait plus ambigu. Revenant au lectorat visé par l'ouvrage, R. Estienne revient également à une visée plus large: " nostre exploict ha son adresse à toute maniere d'enfance, d'aage, de scavoir, ou de tous les deux» [4]. L'expression synthétique finale, "de tous les deux», semble signifier « quels que soient à la fois leur âge et leur degré de savoir. » Par-delà le public spécifique que constitue le latiniste en cours d'apprentissage, le Dictionaire Francoislatin reconquiert donc ici sa pleine utilité pour «tous ceulx qui entendent au faict des lettres » [1]. La notion de jeunesse glisse alors du lecteur à l'ouvrage qui, lui aussi, en est aux balbutiements : " pensant que ce n'est que le pourtraict, et par maniere de dire, l'enfance desdictz livres qui commencent tout premierement à parler.» [4]. Mais dans ce glissement savamment amené (par la reprise du nom "enfance » et la métaphore anthropomorphique), l'on passe du latin au français et de l'apprentissage d'une langue non maternelle à la naissance d'une lexicographie nationale. Il convient de ne pas s'y tromper: si l'image participe ici d'une rhétorique de la modestie de mise dans une préface (l'auteur priant son lecteur d'excuser en cela les possibles imperfections de l'ouvrage : «nous les prions si quelque chose s'y trouve autrement qu'à point, qu'ilz le vueillent excuser :»), elle attire plus encore l'attention sur la valeur de cette entreprise innovante ("tout premierement»), dont le caractère nouveau se conçoit si on l'envisage du point de vue du français.

Dix ans plus tôt, dans son édition du Champ fleury paru en 1529, Geoffroy Tory appelait à cette codification progressive du français :

Toutesfois si nostre langue estoit deument reglée et polie, telles immundices en pourroient estre dejettées. Parquoy je vous prie donnons tous courage les uns aux autres, et nous esveillons à la purifier. Toutes choses ont eu commencement. Quand l'un traictera des lettres, et l'autre des vocales, un tiers viendra, qui declarera les dictions, et puis encores un autre surviendra qui ordonnera la belle oraison. Par ainsi on trouvera que peu à peu on passera le chemin, si bien qu'on viendra aux grans champs poetiques et rhetoriques pleins de belles, bonnes, et odoriferentes fleurs de parler, et dire honnestement et facillement tout ce qu'on vouldra ${ }^{44}$.

21 Après donc G. Tory, qui ouvre la voie en se chargeant de la première étape, celle des lettres (dont il définit le tracé à la règle et au compas), R. Estienne décide de s'occuper des mots (des dictions). Entre temps, il a publié, dès 1531, la Grammatica latinogallica de Sylvius ${ }^{45}$, avant de proposer lui-même, en 1557, une grammaire en français, du fait des critiques qu'avaient pu attirer les premières tentatives de Sylvius et de Meigret, l'un pour les mots picards dont il nourrit son texte, l'autre pour sa tentative de réformation orthographique ${ }^{46}$. Si donc la fin de la préface du Dictionaire rappelle que l'œuvre est destinée à profiter à la « jeunesse » et se clôt sur la notion initiale " d'utilité », elle n'en introduit pas moins, avec une certaine ambiguïté, une nouvelle perspective :

[5] [desdictz livres] Lesquelz, espoir, feront fond le temps advenir de plus riche langaige, mieulx aloyé, et de meilleur coing qu'ilz n'ont à present: au plaisir de 
Jesus Christ, lequel vueille donner accroissement de scavoir à la jeunesse, et perfection à ce que nous avons encommencé à son honneur et à leur utilité.

Avec l'adjectif « riche ", fait ainsi jour, à l'extrême fin de cette première préface, l'idée jusqu'alors différée d'un enrichissement de la langue nationale. Filant toujours le rapprochement entre l'ouvrage et son lectorat, l'auteur pose en regard faire « fond de plus riche langaige, mieulx aloyé, et de meilleur coing » et « donner accroissement de scavoir à la jeunesse ». Ainsi donc, l'utilité de l'ouvrage pour ceux qui apprennent le latin se verra rejaillir paradoxalement sur la langue vernaculaire, amenée à s'enrichir. La clé peut en être donnée dans l'épître « Au Lecteur » de la Grammaire francoise de 1557, laquelle "pourra beaucoup servir principalement à ceulx qui s'aident de nos Dictionaires Latinfrancois, \& Francoilatin, \& s'entremettent de traduire de Latin en Francois $^{47}$ » De la version au thème, l'apprentissage et l'exercice du latin mènent donc à la pratique du français. C'est pourquoi les ouvrages destinés aux latinistes doivent également normer la langue vernaculaire.

\section{La préface de l'édition de 1549}

Cette perspective d'enrichissement du français sur laquelle se terminait la préface de 1539 ouvre celle de 1549. Jean Pruvost ${ }^{48}$ a bien souligné que cette date correspond à la publication de la Deffence, et illustration de la langue francoyse de Du Bellay. Nous pourrions ajouter qu'elle correspond également à la réédition du Champ fleury de G. Tory sous le titre de L'Art et Science de la vraye proportion des lettres Attiques. Si l'on ne trouve point pour autant de manifeste en faveur du français dans cette seconde préface, le discours de l'auteur évolue et les progrès de l'ouvrage suivent ceux de la langue vernaculaire, dix ans après la première édition.

Ces progrès, l'auteur les pose d'emblée, dès l'ouverture du texte : «[1] Oultre la premiere impression de ce present livre, saches Lecteur qu'il a esté en ceste seconde, augmenté d'infiniz mots, ». Cette amplification du contenu, dont B. Quemada montre qu'elle constitue un argument constamment repris dans le discours préfaciel des lexicographes ${ }^{49}$, sera très vite explicitement prise comme témoin de la richesse d'une langue maternelle vivante. Ce n'est pas le cas ici. À ce stade initial, R. Estienne rattache ces progrès à l'entreprise lexicographique elle-même (dont l'enfance poursuit sa croissance pourrait-on dire). Mais si le discours est moins engagé, l'accroissement de l'ouvrage ne s'en trouve pas moins indissociablement lié à la richesse de la langue. La suite de la première phrase suffit à le prouver :

[1] Oultre la premiere impression de ce present livre, saches Lecteur qu'il a esté en ceste seconde, augmenté d'infiniz mots, lesquelz autant que possible a esté, on a tourné en Latin, fors aucuns ausquelz on n'a point encores trouvé és autheurs mots Latins respondans. Pour lesquelz te prions si tu leur trouves propres dictions Latines, de nous en advertir.

24 Si cet extrait est en soi significatif, il le devient plus encore si on le rapproche du passage correspondant de la préface antérieure de 1539 :

L'autre est cestuy cy qui va prenant les motz de la langue Francoise, les mettant après en Latin tout au plus près qu'il s'est peu faire. [4] Et pource que nostre exploict ha son adresse à toute maniere d'enfance, d'aage, de scavoir, ou de tous les deux : nous les prions si quelque chose s'y trouve autrement qu'à point, qu'ilz le vueillent excuser :

25 En 1539, l'auteur donne à entendre qu'il n'a pas toujours été facile de trouver l'équivalent français des mots latins, s'efforçant d'aller «tout au plus près qu'il s'est 
peu faire ». En 1549, l'effort de traduction du français au latin est maintenu «autant que possible ", mais l'empêchement vient désormais du latin dont les auteurs n'ont pu fournir certains équivalents pour les mots français. Si l'humaniste ne saurait reconnaître l'insuffisance de la langue latine, le lexicographe constate les progrès d'une langue vivante sur une langue morte. Ce qui est ici implicite encore deviendra un argument engagé en faveur de la langue nationale chez les lexicographes suivants.

L'évocation des auteurs latins amène («mesmes aussi ») celle des "Rommans et bons autheurs Francois", dont l'auteur dit qu'il a pu omettre de relever certains mots, invitant son lecteur à l'en avertir. C'est là une nouveauté et un progrès considérable. Les études ont montré que R. Estienne avait construit son Thesaurus en dépouillant les auteurs latins qu'il éditait dans ses presses ${ }^{50}$. Rien de tel dans le Dictionaire Francoislatin de 1539 qui se trouvait être le retournement du Dictionarium latino-gallicum, jusque dans le choix des exemples, traduits du latin. L'étape augmentée - enrichie - de 1549 marque donc un progrès dans la manière d'envisager le français et d'en extraire directement un recensement lexical, puisé chez ses propres auteurs et non " tourné » à partir du latin et des Latins.

L'auteur énumère ensuite les ajouts et les nouveautés de cette seconde mouture: "secondement» les mots difficiles ont été expliqués; "tiercement» les noms géographiques ont été insérés. La démarche lexicographique, telle qu'elle est présentée dans la préface, semble désormais orientée du français vers le latin (et non plus du Dictionarium vers le Dictionaire) : les mots géographiques retenus sont ceux qui peuvent être traduits du français en latin ; les mots difficiles incitent les lecteurs à réfléchir sur leur propre langue (et ne servent pas seulement d'adjuvant dans l'apprentissage du latin). En se montrant « contens par ce commencement les avoir seulement incitez de plus près avoir esgard à leur langue " [3], R. Estienne investit son Dictionaire d'un rôle en rapport direct avec le français et revendique sa place aux origines de cette entreprise linguistique nationale.

Certes l'engagement reste encore discret. Si, dans le Thesausus, l'auteur s'étend d'une édition à l'autre sur ses principes de renvois aux auteurs latins ${ }^{51}$ qu'il cite d'après les éditions sorties de ses propres presses, il ne saurait en être question ici pour les auteurs français, qu'il n'édite pas. Il précise en revanche son système de signalement des emprunts à Guillaume Budé (par une lettre B apposée). Dans le paragraphe qu'il lui consacre [5], l'on peut relever quatre évocations du latin, trois du grec et deux du français. Par deux fois, il est rappelé que Budé entendait et écrivait tant le grec que le latin. Mais en posant que «le plus grand nombre des mots Latins bien exposez icy en Francois sont de feu monsieur Budé», pour rappeler ensuite qu'il a eu ce don de comprendre les mots et manières de parler des anciens (" et les Greques bien et proprement expliquer en Latin, Et les Latines en Grec, ou en Francois»), R. Estienne s'inscrit dans une chaîne lexicographique. Après Budé qui a traduit du grec au latin et du latin au grec, le voici qui traduit du latin au français et du français au latin, poursuivant en cela doublement l'œuvre de Budé, parce que celui-ci a également traduit du latin au français et parce que lui-même traduit le latin à partir de Budé. L'intérêt porté au latin mène donc une fois de plus au français selon une sorte de progression inéluctable. Les locutions identifiées par la lettre $B$ sont des formules juridiques provenant des Forensia de Budé52. D'où cette question pertinente de Russon Wooldridge ${ }^{53}$ qui s'interroge sur la présence, en 1549 , de ces «éléments visant le latin de la procédure, surtout que cette langue n'avait en principe plus cours dans le 
domaine de la justice depuis dix ans ». Il y répond en rappelant cette hypothèse de Charles Beaulieux ${ }^{54}$, selon laquelle le Dictionaire de 1539 aurait pu être réalisé sur l'ordre de François $I^{\text {er }}$ afin d'aider les gens de justice habitués à rendre leurs arrêts en latin à passer au français, mais pour la reporter sur l'édition de 1549 qui s'intéresse effectivement à la langue juridique à travers ces formules traduites de Budé.

Il y a là la conscience d'être à la croisée des temps, dépositaire de l'héritage glorieux des Grecs et des Latins et à la naissance d'une destinée linguistique nationale.

[7] Voyla de quoy t'avons voulu advertir, studieux Lecteur, te prians estimer cest ouvrage n'estre que commencement, qui jamais ne se parfera que par diverses personnes soingneuses et diligentes à observer ce que et eulx et autres lisent ou parlent, dont se dressent certaines reigles tant pour l'intelligence des mots, que pour la droicte escripture d'iceulx, comme a esté faict par les autheurs Grecs et Latins.

Comme à la fin de la préface précédente de 1539, l'auteur rappelle donc que l'entreprise n'en est qu'à son commencement. Mais cette fois il y ajoute une comparaison, terminant sur les «autheurs Grecs et Latins » pour inciter, à la suite de G. Tory, les Français à «reigler » leur propre langue. C'est donc désormais vers le français que le regard est tourné et l'ouvrage ne pourra se parfaire qu'en observant ce que les étudits « lisent ou parlent ». Aux Français donc de poursuivre sur cette voie désormais ouverte en suivant l'exemple des Anciens. Avec l'allusion finale aux " autheurs ", R. Estienne change de destinataire. Il ne s'adresse plus cette fois à son lecteur, apprenti latiniste, mais aux auteurs français (ceux-là mêmes peut-être qu'il évoquait à l'ouverture), invités à imiter l'exemple des Grecs et des Latins, pour, comme eux, fixer le sens et l'orthographe des $\operatorname{mots}^{55}$. Il n'est donc pas anodin alors, comme Jean Pruvost l'a souligné ${ }^{56}$, que cette seconde édition augmentée paraisse la même année que la Deffence, et Illustration de la langue françoyse de Du Bellay. Certes le discours n'y est pas aussi engagé, mais un appel aux « autheurs » n'en est pas moins lancé, que Du Bellay portera pour sa part dans une autre direction, s'intéressant aux formes littéraires plutôt qu'au sens et à la graphie. L'entreprise appelée par G. Tory est désormais à l'œuvre. Après donc G. Tory qui règle les lettres en 1529, R. Estienne les dictions en 1539, Du Bellay en vient aux «champs poetiques et rhetoriques» en 1549, en même temps que sont réédités et le traité de Tory et le dictionnaire d'Estienne.

\section{Texte : préfaces du Dictionaire Francoislatin de Robert Estienne (1539 et 1549)}

\section{Principes d'édition}

\section{Textes de référence}

Édition de 1539 : [Robert Estienne], Dictionaire Francoislatin, contenant les motz \& manieres de parler Francois, tournez en Latin. À Paris, De l'imprimerie de Robert Estienne, 1539, Avec privilege du Roy. D'après l'exemplaire en ligne sur le site Gallica (source BnF).

Édition de 1549: [Robert Estienne] Dictionaire Francoislatin, autrement dict Les mots Francois, avec les manieres d'user d'iceulx, tournez en Latin. Corrigé \& augmenté. À Paris, De l'imprimerie de Robert Estienne Imprimeur du Roy, 1549, Par privilege dudict Seigneur. D'après l'exemplaire numérisé sur le site Gallica (source BnF). 


\section{Principes de transcription} suivantes : dissimilation du $i$ et du $j$, du $u$ et du $v$; transcription du $s$ long par un $s$ court et du $B$ par ss; développement des abréviations et de l'éperluette (\& et variantes); introduction de l'apostrophe et désagglutination selon l'usage moderne; distinction

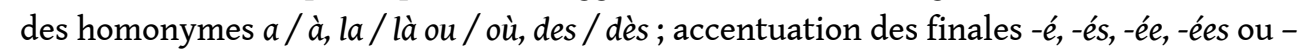
ès (ès, dès, après), l'accentuation n'étant pas introduite en début ou milieu de mot; respect de l'usage des majuscules, y compris après ponctuation moyenne. Afin de faciliter le repérage, nous numérotons les segments du texte.

\section{Liens vers une édition numérisée}

Édition de 1539 de référence numérisée sur Gallica :

http://gallica.bnf.fr/ark:/12148/bpt6k505878.r=.langFR

Édition de 1549 de référence numérisée sur Gallica :

http://gallica.bnf.fr/ark:/12148/bpt6k50589z.r=.langFR

\section{Texte 1 : préface de l'édition de 1539}

[1] Pour ce que la profession de nostr'art nous enhorte à faire tousjours quelque chose qui soit utile en-general à tous ceulx qui entendent au faict des lettres : ou pour le moins duisant aux apprentiz pour lesquelz il fault d'autant plus soigner, qu'ilz ont greigneur besoing de secours, que ceulx qui ont acquiz quelque ruze, et peuvent d'eulx mesmes, sans l'aide d'autruy, passer par les destroictz de la langue Latine : [2] À ceste cause après avoir mis en lumiere le grand Thresor d'icelle langue ${ }^{57}$, lequel peult servir à toutes gens de quelque hault scavoir qu'ilz soyent garniz: nous avons mis cueur et entente au soulagement de la jeunesse Francoise, qui est sur son commencement et bachelage de literature. [3] Si leur avons faict deux livres : L'ung commenceant par les motz Latins deschiffrez en Francois : qui fut publié dès l'année precedente ${ }^{58}$. L'autre est cestuy cy qui va prenant les motz de la langue Francoise, les mettant après en Latin tout au plus près qu'il s'est peu faire. [4] Et pource que nostre exploict ha son adresse à toute maniere d'enfance, d'aage, de scavoir, ou de tous les deux ${ }^{59}$ : nous les prions si quelque chose s'y trouve autrement qu'à point, qu'ilz le vueillent excuser : pensant que ce n'est que le pourtraict, et par maniere de dire, l'enfance desdictz livres qui commencent tout premierement à parler. [5] Lesquelz, espoir, feront fond le temps advenir de plus riche langaige, mieulx aloyé, et de meilleur coing qu'ilz n'ont à present : au plaisir de jesus christ, lequel vueille donner accroissement de scavoir à la jeunesse, et perfection à ce que nous avons encommencé à son honneur et à leur utilité.

Notes lexicales : enhorte : exhorte, incite. entendent au faict des lettres : s'appliquent, se consacrent aux lettres. duisant: instructif. apprentiz: novices. greigneur: plus grand (ancien comparatif synthétique). les destroictz: les difficultés. à cette cause: pour cette raison. après avoir mis en lumiere : après avoir publié. garni : doté, pourvu. mettre entente : effort, soin. bachelage : apprentissage. deschiffré: expliqué, en particulier en parlant de quelque chose d'obscur et de difficile. nostre exploict : notre action (accomplie et menée à terme). ha son adresse : concerne. à point : à propos, comme il se doit. pourtraict : 
esquisse. espoir : peut-être. faire fond: compter, reposer sur. le temps advenir: dans le futur. mieux aloyé : de meilleure qualité (image de l'alliage des métaux).

\section{Texte 2 : préface de l'édition de 1549} ent avoir esté ainsi nommez: ou de quel autre langaige prins et mis en usage Francois : laissans tousjours aux lecteurs leur meilleur jugement, et contens par ce commencement les avoir seulement incitez de plus près avoir esgard à leur langue.

41 [4] Tiercement, ont esté adjoustez les noms des Pais, Isles, Provinces, et Villes, desquelles on a peu trouver les noms Latins correspondans.

[5] Et pourtant que presque en chasque page à la fin de l'explication d'aucuns mots, a esté apposée ceste lettres B, de laquelle ne scaurois ${ }^{61}$ la signification : Sois certain que le plus grand nombre des mots Latins bien exposez icy en Francois sont de feu monsieur Budé $^{62}$, qui sur tous autres, et entre autres dons a eu cestuy de nostre Seigneur, de bien entendre les mots et manieres de parler tant Greques que Latines : et les Greques bien et proprement expliquer en Latin, Et les Latines en Grec, ou en Francois : tellement qu'il n'est memoire de son pareil. [6] Tous ces mots innumerables par luy expliquez nous n'avons maintenant marquez d'un B : ainsi seulement ceulx que depuis sa mort avons transcript d'aucuns de ses livres à nous par ses enfans communiquez d'une singuliere affection (suyvans leur pere) qu'ilz ont de vouloir aider à tous studieux des langues : tant que jamais ne nous ont rien refusé, fust en Grec, ou en Latin, de tout ce que leur pere avoit laissé par escript, qu'ilz ayent estimé à tous povoir prouffiter.

[7] Voyla de quoy t'avons voulu advertir, studieux Lecteur, te prians estimer cest ouvrage n'estre que commencement, qui jamais ne se parfera que par diverses personnes soingneuses et diligentes à observer ce que et eulx et autres lisent ou parlent, dont se dressent certaines reigles tant pour l'intelligence des mots, que pour la droicte escripture d'iceulx, comme a esté faict par les autheurs Grecs et Latins.

Notes lexicales: tourné : traduit (absence d'accord du participe passé avec le complément antéposé) ; fors : excepté ; aucuns : certains, quelques-uns (sens positif en contexte négatif); és: dans les; respondans: correspondant; propre diction: mot approprié ; conception : idée, projet ; quelque fois : un jour (quelconque à venir); sortir son effect : s'accomplir, se réaliser ; pourtant que : parce que ; innumerables : innombrables; $a$ tous studieux des langues : à tous ceux qui étudient les langues; dont : d'où ; intelligence : compréhension. 


\section{Bibliographie de corpus}

\section{Éditions anciennes}

1539 - [ESTIENNE, Robert], Dictionaire Francoislatin, contenant les motz \& manieres de parler Francois, tournez en Latin. À Paris, De l'imprimerie de Robert Estienne, 1539, Avec privilege du Roy in-fol. (achevé d'imprimé 1540). DEU, Augsburg, Staats- und Stadtbibliothek [2.Spw.42]. DEU, München, Bayerische Staatsbibliothek [2.L.lat.f.29]. DEU, München, Bibliothek der Ludwig-MaximiliansUniversität [0001/2.Philol.152]. DEU, Zürich, Zentralbibliothek, Alte Drucke [réserve livres anciens] [RRc.22]. FRA, Avignon, BM, fonds patrimoniaux [Folio 2283] ${ }^{63}$. FRA, Laon, BM [in4.B.82]. FRA, Lyon, BIU (Bibliothèque InterUniversitaire)-LSH [2937/2]. FRA, Paris, BnF, Arsenal [FOLBL-284]. FRA, Paris, BnF, Tolbiac [RES-G-X-36]. FRA, Paris, EPHE-4 [GP.13]. FRA, Paris, Institut de France [Fol.0.55.B**]. FRA, Paris, Université Paris-4 Sorbonne, BUFR littérature française et comparée, [Res.1088]. FRA, Paris, Université Paris-4 Sorbonne, BU [RXVI.458 pièce 2]. Communication différée. FRA, Tours, BU Lettres [FB1972]. FRA, Troyes, BM [t.3.61-Belles Lettres]. FRA, Verdun, Bibliothèque d'étude Codecom [74569 $]^{64}$. FRA, Vesoul, BM [l'exemplaire a disparu] ${ }^{65}$. GBR, Cambridge, Sidney Sussex College, Muniment Room [A.4.21]. GBR, Cambridge, University Library, Rare Books Room [Peterborough.B.7.25]. GBR, Manchester, Deansgate [Christie Coll. / 22.k.7(2)]. GBR, Newcastle, Robinson Library, Special Collections [SSBE St Bees 151]. GBR, Peterborough Cathedral Library [cote ?]. ITA, Bologne, Biblioteca universitaria di Bologna [Tab. 4.E.2.10]. PRT, Biblioteca Nacional de Portugal [L.2882.A].

1549 - [ESTIENNE, Robert], Dictionaire Francoislatin, autrement dict Les mots Francois, avec les manieres d'user d'iceulx, tournez en Latin. Corrigé \& augmenté. À Paris, De l'imprimerie de Robert Estienne Imprimeur du Roy, 1549, Par privilege dudict Seigneur, in-fol., 675 p. FRA, Abbeville, BM [FA.16.C. 74]. FRA, Ajaccio, BM [25.XLVIII.4], fonds patrimonial. FRA, Arles, Médiathèque [BB.3470.M fonds ancien]. FRA, Avignon, BM, fonds patrimoniaux [Folio 2284]. FRA, Beaune, BM [C 372-16 ${ }^{\mathrm{e}}$ siècle]. FRA, Macon, BM [1941-Havard]. FRA, Nice, BMVR Louis Nucéra [XVI-1608]. FRA, Nîmes, Bibliothèque Carré d'Art [24309-16e siècle]. FRA, Paris, BnF [X-592]. FRA, Paris, École Normale Supérieure, Ulm, réserve [LP.1.8.f ${ }^{\circ}$. FRA, Paris, Institut de France [Fol.0.55.B]. FRA, Paris, Museum d'Histoire Naturelle [Y2.1230]. FRA, Rennes, BMVR, Rennes-Métropole [648-16 ${ }^{\mathrm{e}}$ siècle]. FRA, Rodez, Médiathèque [RES.15645]. FRA, Strasbourg, BNUS [CD.10.846]. FRA, Strasbourg, Strasbourg-2 BU Portique [DICT/83]. FRA, Tours, BU Lettres [FB.1973], fonds Brunot. FRA, Troyes, BM [t.3.59 - Belles Lettres]. GBR, Cambridge, University Library, Rare Books Room [Bb*.2.8(C)]. GBR, Cambridge, Trinity College, Lower Library [C.15.1]. GBR, Durham Cathedra Library [cote ?]. GBR, Durham, Palace Green Library, Bamburgh [Bamburgh N.1.23]. GBR, Oxford, St John's College Library [Omega.4.40]. GBR, Oxford, Taylor Institution Main Library [ARCH.FOL.F.1549]. Confined. ITA, Modena, Biblioteca Estense Universitaria [A.006.0.026]. ITA, Padova, Biblioteca del Seminario Vescovile [500.FORC.0.3.12]. ITA, Roma, Biblioteca nazionale centrale Vittorio Emanuele II [68.2.E.3]. ITA, Roma, Biblioteca dell'Accademia nazionale dei Lincei e Corsiniana [CORS.V.B.15]. SVN, Ljubljana, Narodna in univerzitetna knjižnica [6010]. 


\section{Éditions modernes}

Estienne, Robert, Dictionnaire françois-latin [1549], Genève, Slatkine, diff. : Paris, Champion, 1972. Reproduction fac-similé.

Estienne, Robert, Dictionnaire françois-latin [1549], Paris, France-Expansion, 1972. Reproduction microforme (22 microfiches).

Estienne, Robert, Dictionnaire francois latin [1539], Paris, France-Expansion, 1973. Reproduction microforme (17 microfiches).

CD-ROM : Le grand atelier historique de la langue française : l'histoire des mots du Haut Moyen-Age au $X I X^{e}$ siècle : 14 grands dictionnaires de la langue française, présenté par Isabelle Leroy-Turcan, Marsanne, Redon, 2001 (rééd. 2002).

Édition électronique : Grand Corpus des dictionnaires [du $9^{e}$ au $20^{e}$ siècle], Paris, Éditions Garnier Numériques.

Base en ligne : Early Dictionary Databases / Informatique et dictionnaires anciens, éd. I. Lancashire, T. R. Wooldridge. Base en ligne : http://projects.chass.utoronto.ca/chwp/

\section{Études critiques}

Soulignons ici la belle initiative scientifique de Russon Wooldridge qui, dans le cadre du laboratoire CHASS de l'université de Toronto, a publié en version électronique, accessibles en ligne, les nombreuses études qu'il a consacrées aux débuts de la lexicographie française. AQUILON, Pierre, Estienne (Robert) (1520-1559), Centurice latince. Cent une figures humanistes de la Renaissance aux Lumières offertes à Jacques Chomarat, dir. Colette Nativel, Genève, Droz, 1997, p. 351-354.

ARMSTRONG Elizabeth, Robert Estienne Royal Printer. An Historical Study of the Elder Stephanus, Cambridge, University Press, 1954. Rééd. 1986.

BEAULIEUX, Charles, « Liste des dictionnaires, lexiques et vocabulaires français antérieurs au Thresor de Nicot (1606) », Mélanges de philologie offerts à F. Brunot, Paris, Société Nouvelle de Librairie et d'Édition, 1904, p. 371-398.

BEAULIEUX, Charles, « Les dictionnaires de la langue française du seizième siecle », Revue internationale de l'enseignement, mai-juin 1927, p. 155-167.

BEAULIEUX, Charles, « Dictionnaire », in Dictionnaire des lettres françaises, t. I, dir. G. Grente, Paris, Fayard, 1951.

BRANDON, Edgar E., Robert Estienne et le dictionnaire français au XVIe siècle, Baltimore, J. H. Furst, 1904. Rééd. : Genève, Slatkine Reprints, 1967.

BRAY, Laurent, « La lexicographie française des origines à Littré », in HAUSMANN et al., Wörterbücher : Ein internationales Handbuch zur Lexicographie / Dictionnaires : Encyclopédie internationale de lexicographie, Berlin \& New York, Walter de Gruyter, 1989-1991 (t. I, 1989 ; t. II, 1990 ; t. III, 1991), p. 1788-1818.

BURIDANT, Claude, « Lexicographie et glossographie médiévales. Esquisse de bilan et perspectives de recherche ", Lexique, 4, 1986, p. 9-46. 
CATACH, Nina, Les Modifications orthographiques des dictionnaires de l'Académie française (1694-1935) précédées de certaines graphies comparatives des Dictionnaires de R. Estienne (1549) et J. Nicot (1606), thèse manuscrite, 1968.

CATACH N., GOLFEND J., METTAS O., PASQUES L., R. E. N. A., Dictionnaire historique de l'orthographe française (abrégé R. Estienne, Nicot, Académie), Fascicule d'essai dactylographié, 1976.

CNRS-AUPELF, Archives de la linguistique française, collection de documents relatifs à la langue française, publiés entre 1500 et 1900, micro-réédition sous la dir. de B. Quemada, Paris, France-Expansion, 1972.

CRAPELET, G. A., Robert Estienne, imprimeur royal, et le roi François Ier. Nouvelles recherches sur l'état des lettres et de l'imprimerie au XVI siècle, Paris, 1839.

DUTKO, Zoltan-E, « Contribution chronologique à l'étude du vocabulaire du XVI siècle ", Français moderne, oct. 1935, p. 356-362.

FURNO, Martine, « À l'aube de la bibliographie : les références externes dans les dictionnaires latins, 1480-1545 », Réforme, Humanisme, Renaissance, vol. 34, nº 3 (2011), p. 47-63.

FURNo, Martine, «Qui et Que dans le Dictionnaire François-Latin de Robert Estienne », Langue Française, 139, septembre 2003 : La grammatisation du français : qui que quoi vs qui(s) quod entre XVI et XVII siècles, dir. B. Colombat, p. 28-46.

FURNO, Martine, « Statut des termes du métalangage grammatical dans les dictionnaires monolingues latins et bilingues latin-français (XII ${ }^{\mathrm{e}}-\mathrm{XVIII}{ }^{\mathrm{e}}$ siècles) ", Métalangage et terminologie linguistique. Actes du colloque international de Grenoble (Université Stendhal - Grenoble III, 14-16 mai 1998), dir. B. Colombat et M. Savelli, Leuven/Paris/Sterling, Virginia, Peeters, 2001, p. 331-347. FURNo, Martine, «Le mariage de Calepin et du Thesaurus, sous l'olivier de Robert Estienne, à Genève, en 1553 ", Bibliothèque d'Humanisme et Renaissance, 63, 2001, nº 3, p. 511-532. FURNO, Martine, « Les dictionnaires de Robert Estienne : sens et finalités d'une œuvre lexicographique », Voces, 10-11, 1999-2000, p. 11-27.

FURNO, Martine, « Les lemmes grammaticaux dans les dictionnaires latins au début du XVI ${ }^{\mathrm{e}}$ siècle : l'exemple de Robert Estienne ", El Brocense y las humanidades en el siglo XVI, Salamanca, Ediciones Universidad, 2003, p. 169-189.

FURNo, Martine, « Doctrina vel disciplina. Tensions et évolutions des dictionnaires latins humanistes, 1502-1636 », Les Instruments de travail à la Renaissance, dir. J.-F. Gilmont et A. Vanautgærden, Brespols, 2010 , p. 69-103.

FURNo, Martine, « De la métaphore à la standardisation : l'intitulé des dictionnaires du latin et du français, 1500-1650 », Le français préclassique, 10, 1500-1650, 2007, p. 171-181.

La France des humanistes. Robert et Charles Estienne. Des imprimeurs pédagogues, éd. Bénédicte Boudou, Judit Kecskeméti, Turnhout, Brepols, 2009.

LECOINTRE, Simone ; LE GALLIOT J., « Le lexique dans l'histoire : problèmes et perspectives », Langue française, 10, mai 1971, p. 57-82.

LINDERMANN, Margarete, « Le Vocabularius Nebrissensis latin-français et les débuts de la lexicographie française ", dans Actes $d u I V^{e}$

colloque international sur le Moyen Français, éd. Anthonij Dees, Amsterdam, Rodopi, 1985, p. 55-86. MARTIN, Henri-Jean, « Le temps de Robert Estienne », Histoire de l'édition française, vol. 1, Paris, 1982, p. 230-235. 
QUEMADA, Bernard, Les Dictionnaires du français moderne, 1539-1863, Paris/Bruxelles/Montréal, Didier, 1967.

QUEMADA, Bernard, Essai de bibliographie générale des dictionnaires, vocabulaires et glossaires français, Besançon, Centre d'Étude du Vocabulaire français, 1967.

PRUVOST, Jean, Les Dictionnaires de langue française, Paris, PUF, « Que sais-je ? », 2002.

RENOUARD, Antoine A., Annales de l'imprimerie des Estienne, Paris, Jules Renouard, 1843. Réimpr. : New York, B. Franklin, 1960.

STARNES, Dewith T., Robert Estienne's Influence on Lexicography, Austin, University of Texas Press, 1963.

TORO, Michel de, «À propos du dictionnaire français-latin de Robert Estienne », Français moderne, octobre 1936, p. 354-360.

WOOLDRIDGE, Russon, « Robert Estienne, cruciverbiste : les équations sémantiques du Dictionaire francoislatin ", Cahiers de lexicologie, 27, 1975-II, p. 107-116. http://homes.chass.utoronto.ca/ $\sim$ wulfric/nicot/cruci75/

WOOLDRIDGE, Russon, « Pour une exploration du français des dictionnaires d'Estienne et de Nicot (1531-1628) », Français moderne 46, juillet 1978, p 210-225. http://homes.chass.utoronto.ca/ $\sim$ wulfric/articles/fr-mod/

WOOLDRIDGE, Russon, « Les sources des dictionnaires français d'Estienne et de Nicot », La Langue française au XVI siècle : usage, enseignement et approches descriptives (éd. P. Swiggers \& W. van Hœcke), Louvain-Paris: Peeters, 1989, p. 78-99. http://homes.chass.utoronto.ca/ wulfric/nicot/ sources/

WOOLDRIDGE, Russon, « Le FEW et les deux millions de mots d'Estienne-Nicot : deux visages du lexique français », Travaux de Linguistique et de Philologie, 28, 1990, p. 239-316. http:// homes.chass.utoronto.ca/ wulfric/articles/few_90/

WOOLDRIDGE, Russon, « Structures du Corpus et de la Base Estienne-Nicot (1531-1628) », CCH Working Papers, 2, 1992, p 21-32. http://projects.chass.utoronto.ca/chwp/tiden/http:// projects.chass.utoronto.ca/chwp/tiden/

WOOLDRIDGE, Russon (éd.), Historical Dictionary Databases, CCH Working Papers, 2, 1992. http:// projects.chass.utoronto.ca/chwp/

WOOLDRIDGE, Russon, «Le lexique français de la Renaissance dans les textes et dans les dictionnaires », Actes du XXIe Congrès de linguistique et philologie romanes, Palerme, 18-23 septembre 1995, Tübingen, Niemeyer, 1996. http://homes.chass.utoronto.ca/ wulfric/articles/pal995/

WOOLDRIDGE, Russon, « Vitruve latin et français dans les dictionnaires de Robert Estienne », publié dans les Actes du Colloque sur la traduction et l'adaptation en France à la fin du Moyen Âge et à la Renaissance (Nancy II, mars 1995). http://homes.chass.utoronto.ca/ wulfric/articles/nan395/ WOOLDRIDGE, Russon, Les Débuts de la lexicographie française. Etienne, Nicot et le Thresor de la langue françoyse (1606), University of Toronto Press, 1977.

Réédition électronique : Edicta, 1997 ; Toronto, Le Net des Études françaises, 2010 :

http://homes.chass.utoronto.ca/ wulfric/edicta/wooldridge/

http://www.etudes-francaises.net/dossiers/wooldridge_debuts/ 
WOOLDRIDGE, Russon, Base de pièces préfatoires interactives (base de données contenant de nombreuses préfaces de dictionnaire, d'Estienne 1539 à Académie 1932) : http:// www.chass.utoronto.ca/ wulfric/naf/prefaces/

\section{ANNEXES}

\section{La place du français dans l'œuvre de Robert Estienne}

Tableau des ouvrages publiés par Robert Estienne, établi d'après : Antoine Augustin Renouard, Annales de l'imprimerie des Estienne, Paris, Jules Renouard et C ${ }^{\text {ie }}, 1838$, première partie : «Robertus Stephanus Primus ", p. 25-96 (ouvrage numérisé sur Google Books). Pour un recensement bibliographique complet, l'on pourra également consulter : La France des Humanistes. Robert et Charles Estienne. Des imprimeurs pédagogues, éd. B. Boudou et J. Kecskeméti, Turnhout, Brepols, 2009.

\begin{tabular}{|c|c|c|}
\hline année de publication & ouvrages publiés & ouvrages en français \\
\hline 1526 & 9 & 1 \\
\hline 1527 & 16 & \\
\hline 1528 & 19 & 1 \\
\hline 1529 & 25 & \\
\hline 1530 & 14 & \\
\hline 1531 & 11 & \\
\hline 1532 & 13 & \\
\hline 1533 & 15 & \\
\hline 1534 & 16 & \\
\hline 1535 & 17 & 1 \\
\hline 1536 & 22 & \\
\hline 1537 & 22 & \\
\hline 1538 & 8 & \\
\hline 1539 & 13 & 1 \\
\hline 1540 & 13 & 2 \\
\hline 1541 & 15 & \\
\hline 1542 & 13 & \\
\hline 1543 & 14 & 2 \\
\hline 1544 & 28 & 5 \\
\hline 1545 & 17 & 1 \\
\hline 1546 & 24 & \\
\hline 1547 & 23 & 4 \\
\hline 1548 & 18 & \\
\hline
\end{tabular}




\begin{tabular}{|l|l|l|}
\hline 1549 & 22 & 3 \\
\hline 1550 & 16 & 1 \\
\hline 1551 & 9 & 4 \\
\hline 1552 & 8 & 3 \\
\hline 1553 & 8 & 1 \\
\hline 1554 & 12 & \\
\hline 1555 & 5 & 2 \\
\hline 1556 & 2 & 1 \\
\hline 1557 & 7 & 1 \\
\hline 1558 & 6 & 1 \\
\hline 1559 & 5 & $35(6.86 \%)$ \\
\hline 1560 & 2 & \\
\hline éd. posthume & 23 & \\
\hline sans date & 510 & \\
\hline & & \\
\hline
\end{tabular}

D'après cette même source, les ouvrages publiés en français sont (par ordre chronologique) les suivants :

1. La maniere de tourner en langue Francoise les Verbes actifz, passifz, Gerundifz, Supins et Participes; Item les verbes Impersonnelz aianzs terminaison active ou passive, avec le verbe substantif nommé sum, Paris, Robert Estienne, 1526. Réédition en 1528, puis en 1535. Une édition « reveue \& corrigée en grande diligence » paraît en 1547.

2. Dictionaire Francoislatin, contenant les mots et manieres de parler Francois tournez en Latin, Paris, Robert Estienne, 1539. Nouvelle édition : Dictionaire «Corrigé \& augmenté », 1549.

3. Decem prcecepta, \& Scripturce Summa, Et les Dix Commandements, avec l'Extrait abrégé de l'Ecriture, imprimés chacun en placard sur une feuille in fol. ouverte, Robert Estienne, 1540.

4. Des coustumes et manieres de vivre des Turcs, faict en latin par Christophe Richer, \& par iceluy Richer traduict en langue Françoise, Paris, Robert Estienne, 1540.

5. Translation de l'Epistre du roy treschrestien François premier de ce nom, à nostre sainct Pere Paul troisieme, par laquelle est respondu aux calomnies contenues en deux lettres envoyées au dict sainct Pere par Charles cinqiesme empereur, Paris, Robert Estienne, 1543. Deux éditions la même année : in- $4^{\circ}$ et in- $8^{\circ}$.

6. Les mots francois selon l'ordre des lettres, ainsi que les fault escrire, tournez en latin pour les enfants, Paris, Robert Estienne, 1544. Réédité en 1547. Nouvelle édition en 1557 sous le titre : Dictionaire des mots françois selon l'ordre des lettres, ainsi que les fault escrire; avec les manieres de parler plus nécessaires ; tournez en latin et amplifiez de beaucoup pour l'utilité des enfants et autres, s. 1., Robert Estienne, 1557.

7. Hecuba d'Euripide, traduicte de grec en Rhythme françoise [par Lazare de Baïf], dediée au Roy, Paris, Robert Estienne, 1544. Réédition en 1550 sous le titre : La Tragedie d'Euripide, nommée Hecuba, traduicte de grec en rhythme Françoise par Lazare de Bayf, dediée au Roy. 
8. Messire Jean de Bellay cardinal. Harangues, Oraisons, Epistres, \& autres choses tant en Latin qu'en François, Paris, Robert Estienne, 1544.

9. Response à une epistre envoyée de Spire par ung secretaire alemand à ung serviteur du Roy treschrestien. Aultre epistre des choses faictes depuis quatre ans en Europe, Paris, Robert Estienne, 1544.

10. Defence pour le Roy de France Treschrestien, à l'encontre des injures \& detractions de Iaques Omphalius, faicte nagueres en Latin par ung serviteur du Roy, \& maintenant traduicte en François, par Simon Brunel, Paris, Robert Estienne, 1544.

11. Les Declinaisons des noms et verbes que doibvent scavoir entierement par cueur les enfants, ausquelz on veult bailler entrée à la langue latine. Ensemble la maniere de tourner les noms, pronoms, verbes, tant actifs que passifs, gerondifs, supins \& participes : les verbes Sum, Vole, Nolo, Malo, Fero, Edo es, Fio, Possum, Memini : aussi les impersonelz. Des huicts parties d'oraison. La maniere d'exercer les enfants à décliner les noms et les verbes, Paris, Robert Estienne, 1545. Réédité en 1549.

12. Le Trespas, Obseques et enterrement de treshault, trespuissant, et tresmagnanime François par la grace de Dieu, Roy de France, treschrestien, premier de ce nom, prince clement, pere des arts et sciences. Les deux Sermons funebres prononcez esdictes obseques, l'ung à Nostre Dame de Paris, l'autre à Sainct Denis en France, par Pierre Chastelain, Paris, Robert Estienne, $1547 / 1548$.

13. Oraison funebre de François I, contenant un brief discours de ses gestes, facits et actions les plus remarquables ; traduite du latin de Pierre Du Chastel, par Jean Martin, Paris, Robert Estienne, 1547.

14. Les Principes et premiers Elemens de la langue latine, par lesquels tous jeunes enfants seront facilement introduicts à la cognoissance d'icelle, Paris, Robert Estienne, 1549.

15. Pseaumes de David, en Latin \& François, s. 1., Robert Estienne, 1552.

16. Sommaire et brefs recueils en forme d'exposition du contenu ès Pseaumes de David, s. 1., Robert Estienne, 1552.

16. Le Nouveau Testament, c'est-à-dire la Nouvelle Alliance de Nostre Seigneur Jesus-Christ, tant en latin qu'en françois. Les deux Translations traduites du grec, respondantes l'une à l'autre verset à verset, notez par nombres. Brieve declaration d'aucuns mots \& manieres de parler contenus en iceluy, difficiles à entendre. Claire declaration du contenu au Vieil \& Nouveau Testament. Sommaires \& briefs recueils en forme d'exposition du contenu ès chapitres de tous les livres du Nouveau-Testament, s. 1., Robert Estienne, 1552.

17. Les Censures des Theologiens de Paris, par lesquelles ils avoyent faulsement condamné les Bibles imprimées par Robert Estienne, Imprimeur du Roy : avec la response d'iceluy Robert Estienne. Traduictes de Latin en Francois, s. 1., Robert Estienne, 1552.

18. La Bible. Traduction françoise revue par Jean Calvin, s. l., Robert Estienne, 1553.

19. Catechisme, par Jehan Calvin, s. 1., Robert Estienne, 1553.

20. La forme des prieres Ecclesiastiques, avec la maniere d'administrer les Sacrements, \& celebrer le mariage, \& la visitation des malades, s. l. , Robert Estienne, 1553.

21. Les Quatre Evangelistes, avec une Exposition continuelle \& familiere, recueillie des expositions des plus sçavans docteurs Ecclesiastiques, par laquelle on peut voir combien les 
Gloses ordinaires \& Postilles, que le temps passé on a baillé au peuple chrestien en lieu de l'Evangile, l'ont esloingné \& d'estourné de Jesus-Christ, \& en quelles tenebres on l'a mené, s. l., Robert Estienne, 1554.

22. Traicté de la Grammaire francoise, s. 1., Robert Estienne, 1557. Rééditée en 1558.

23. Le Nouveau Testament, reveu et corrigé sur le grec par l'avis des ministres de Geneve, s. 1., Robert Estienne, 1560. Édition commencée par Robert Estienne, achevée et publiée après sa mort.

24. Alphabeth hebreu, s. 1., s. n., s. d.

\section{NOTES}

1. E. E. Brandon (Robert Estienne et le dictionnaire français au XVI ${ }^{e}$ siècle, Baltimore, J. H. Furst, 1904 ; rééd.: Genève, Slatkine Reprints, 1967, p. 6 et p. 25 n. 2) explique que «tous ses biographes" retiennent la date de 1503 en se fondant sur l'autorité de Théodore de Bèze (Icones id est Verce Imagines virorum doctrina simul et pietate illustrium, Ioannes Laonius, 1580) qui le dit âgé de 56 ans à sa mort en septembre 1559. Il préfère pour sa part le témoignage de Henri II, fils de Robert Estienne, porté sur la feuille commémorative qu'il édite en l'honneur de son père et dans laquelle il précise: "Vixit ann. LIX men. XI", ce qui amène E. E. Brandon à proposer une naissance en octobre 1498. Cette proposition se heurte toutefois à la date du mariage de Henri Estienne père et de Guyonne Viart, veuve de l'imprimeur Jean Higman, dont des publications portent le nom jusqu'en 1500, ce qui ferait situer le mariage en 1500 ou 1501. E. E. Brandon rappelle cet argument (op. cit., p. 3, n. 3 et p.6), mais suggère que les associés de J.Higman auraient pu afficher son nom quelque temps encore après sa mort.

2. P. Aquilon, «Estienne (Robert) (1520-1559) », Centurice latince. Cent une figures humanistes de la Renaissance aux Lumières offertes à Jacques Chomarat, dir. Colette Nativel, Genève, Droz, 1997, p. 351-354, spé. p. 351.

3. P. Aquilon, art. cité, p. 351.

4. Huit seulement selon P. Renouard (Annales de l'imprimerie des Estienne, Paris, Jules Renouard, 1843, p. 35) ; neuf selon E. E. Brandon (op. cit., p. 13).

5. D'après E. E. Brandon (ibid., p. 13), sa première épouse serait morte en 1546 ou 1547.

6. Selon M. Furno qui précise qu'ils ont été déshérités pour « retour au papisme " ( Robert I Estienne imprimeur ", dans La France des Humanistes. Robert et Charles Estienne. Des imprimeurs pédagogues, éd. B. Boudou et J. Kecskeméti, Turnhout, Brepols, 2009, p. 22). A. Renouard (op. cit., p. 34) ne mentionnait que le cas du second fils Robert, établi à Paris.

7. Premier volume de La Bibliotheque du sieur de la Croix du Maine. Qui est un catalogue general de toutes sortes d'Autheurs, qui ont escrit en François depuis cinq cents ans et plus, jusques à ce jourd'huy, [...], À Paris, Chez Abel l'Angelier, 1584, p. 443.

8. P. Virgilii Maronis Opera. Mauri Servii Honorati Grammatici in eadem Commentarii ex antiquis exemplaribus suce integritati restituti.

9. M. Furno, « Robert I Estienne imprimeur », éd. citée, p. 21.

10. Il ne reste plus de trace de ces placards que R. Estienne évoque dans son introduction à sa «Réponse aux censures des Théologiens de Paris ( voir infra note 12). La Summa a été également publiée en tête de plusieurs éditions de la Bible $(1540,1545,1546, \ldots)$.

11. Le Collège Royal ou Collège des lecteurs royaux a pris, en 1870, le nom de Collège de France, qu'il conserve aujourd'hui.

12. Les Censures des Theologiens de Paris, par lesquelles ils avoyent faulsement condamné les Bibles imprimées par Robert Estienne, Imprimeur du Roy : avec la response d'iceluy Robert Estienne. Traduictes 
de Latin en Francois, [Genève], Robert Estienne, 1552. L'ouvrage a été également publié en latin la même année.

13. Les lettres patentes qui le décrètent sont signalées dans le Catalogue des Actes de François $I^{\text {er }}$ (BnF, Ms. Fr 21816, VIII, n³2840, p. 683), mais demeurent introuvées. E. Armstrong signale toutefois des privilèges (celui des Forensia de Budé ou des Mots francois de 1544) évoquant explicitement ces lettres-patentes (Elizabeth Armstrong, Robert Estienne Royal Printer. An Historical Study of the Elder Stephanus, Cambridge, University Press, 1954, p. 37-38).

14. Selon E. Armstrong, op. cit., p. 121.

15. E. Armstrong (ibid., p. 124-125) la relève pour sa dernière occurrence dans le colophon daté du 18 avril 1542 du De Puella quae sine cibo et potu vitam transigit, brevis narratio de Gerardus Bucoldianus (Paris, Estienne, 1542).

16. P. Aquilon, art. cité, p. 352.

17. Sur cette édition: M. Furno, «Robert Estienne et les Pères de l'Église : quelques remarques sur l'édition d'Eusèbe de Césarée (1544) », L'Antiquité en ses confins, mélanges offerts à Benoît Gain, I numéro hors-série de Recherches \& Travaux, 16, 2008, p. 85-94. Et pour l'histoire de ces " grecs du roi » emportés par R. Estienne à Genève et rachetés ensuite par la France : P. Renouard, op. cit., p. 36-44.

18. M. Roques, Recueil général des lexiques français du Moyen Âge (XII ${ }^{e}-X V^{e}$ siècle), I: lexiques alphabétiques, Paris, Champion, 1936 (I)-1938 (II). L'abavus est reproduit dans le tome I d'après cinq manuscrits : Douai, Évreux, Paris-BnF, Vaticane, Conches. Ce premier mot figure dans les trois premiers manuscrits, le début manquant dans les deux derniers. Le ms. de Douai (BM, $\mathrm{n}^{\circ} 62$ [ancien 82 et 109] est le plus ancien du groupe. M. Roques (p. XVII) le date de la fin du XIII ${ }^{\mathrm{e}}$ siècle. 19. E. E. Brandon, op. cit., p. 28.

20. M. Lindermann, « Le Vocabularius Nebrissensis latin-français et les débuts de la lexicographie française", dans Actes du IV colloque international sur le Moyen Français, éd. Anthonij Dees, Amsterdam, Rodopi, 1985, 6), p. 55-86, spé. p. 64 sq.

21. Si E. E. Brandon donne crédit à ce récit préfaciel (op. cit., p. 30-31), il n'en reconnaît pas moins en note (p. 31, n. 2) qu'une révision n'était pas pour autant impossible, à preuve la publication du Calepin révisé par R. Estienne en 1553: "Mais en 1528 il aurait été plus difficile de revoir le "Calepin" et d'en faire un dictionnaire tel que Robert Estienne rêvait que de compiler une œuvre nouvelle. C'est donc en ce sens qu'il faut interpréter le récit de la préface. »

22. H. Estienne, Thesaurus græece linguce, Genève, Henri Estienne, 1572, 5 vol.

23. Op. cit. , p. 443-444.

24. La Bibliotheque d'Antoine du Verdier, seigneur de Vauprivas, Contenant le Catalogue de tous ceux qui ont escrit, ou traduict en François, \& autres Dialectes de ce Royaume, [...], À Lyon, par Barthelemy Honorat, 1585, p. 271, article « Dictionaire».

25. L'on peut citer toutefois le Catholicon du prêtre breton Jean Lagadeux, qui part des mots bretons et y ajoute une définition française et un équivalent latin. M. Lindermann le signale comme « le plus ancien lexique polyglotte » (art. cité p. 63), précisant que la BnF en possède un manuscrit approximativement daté de 1464 et qu'il fut ensuite imprimé en 1499.

26. B. Quemada, Les Dictionnaires du français moderne 1539-1863, Paris, Didier, 1967, p. 46.

27. J. Pruvost, Les Dictionnaires de langue française, Paris, PUF, «Que sais-je ?», 2002, p. $6:$ «À l'origine, un dictionaire bilingue ».

28. «Mais le destin qui a fait du Dictionnaire françois-latin le point de départ d'une tradition qui court ensuite jusqu'au Dictionnaire de l'Académie et à Littré, a aussi éclairé le travail d'Estienne d'une lumière faussée. Quels qu'aient été les enrichissements et les améliorations dont il a bénéficié dans la seconde édition, le lexique d'Estienne est en effet plus le point d'aboutissement d'une œuvre latine [entreprise avec la publication du Thesaurus Linguce latince en 1531] que le début d'une œuvre vernaculaire.» M. Furno, "Qui et Que dans le Dictionnaire François-Latin de 
Robert Estienne ", Langue Française, 139, septembre 2003: La grammatisation du français: qui que quoi vs qui(s) quod entre XVI et XVII e siècles, dir. B. Colombat, p. 28-46, spé. p. 28.

29. Dans le Thesaurus, M. Furno estime à plus de $90 \%$ la part du latin, soit moins de $10 \%$ de français, lequel est réservé, ponctuellement, à un éclaircissement dans le cas de mots techniques (par ex. nom de plante ou d'animaux) ou d'expressions idomatiques qu'un lecteur moyen ne peut pas encore saisir directement en latin. M. Furno, «Doctrina vel disciplina. Tensions et évolutions des dictionnaires latins humanistes, 1502-1636", Les Instruments de travail à la Renaissance, dir. J.F. Gilmont et A. Vanautgærden, Brespols, p. 69-103, spé. p. 89.

30. M. Furno, «Qui et Que dans le Dictionnaire François-Latin de Robert Estienne », éd. citée, p. 29.

31. M. Furno, « Doctrina vel disciplina. Tensions et évolutions des dictionnaires latins humanistes, 1502-1636 », éd. citée, p. 73.

32. M. Furno, «Les dictionnaires de Robert Estienne: sens et finalité d'une œuvre lexicographique », Voces, 10-11, 1999-2000, p. 11-27, spé. p. 25.

33. Selon la base étymologique du Centre National de Ressources Textuelles (CNRTL), le terme est emprunté au latin médiéval dictionarium (attesté dès 1220 avec le Dictionarius de J. De Garlande) et dérive du latin dictio (-onis) " action de dire, propos, mode d'expression ». Sa première attestation en français remonte au début du XVI $\mathrm{X}^{\mathrm{e}}$ siècle (A. Vérard, Le Jardin de Plaisance, $\mathrm{f}^{\circ}$ [a V vº, 1501 , cité par B. Quemada, Fichier).

34. M. Furno, « De la métaphore à la standardisation : l'intitulé des dictionnaires du latin et du français, 1500-1650 », Le français préclassique, 10, 1500-1650, p. 171-181, spé. p. 174-178.

35. J. Pruvost, op. cit., p. 20-23 (spé. p. 20) pour l'histoire de ces titres.

36. M. Furno, «À l'aube de la bibliographie : les références externes dans les dictionnaires latins, 1480-1545 », Réforme, Humanisme, Renaissance, vol. 34, n³ (2011), p. 47-63, note 14.

37. M. Furno, « Doctrina vel disciplina. Tensions et évolutions des dictionnaires latins humanistes, 1502-1636 » éd. citée, p. 86.

38. M. Furno, «Qui et Que dans le Dictionnaire François-Latin de Robert Estienne », éd. citée, p. 29.

39. C. Beaulieux, «Liste des dictionnaires, lexiques et vocabulaires français antérieurs au Thresor de Nicot (1606) ", Mélanges de philologie offerts à F. Brunot, Paris, Société Nouvelle de Librairie et d'Édition, 1904, p. 371-398, spé. p. 371.

40. Cit. et trad. M. Furno : «En effet, quoi de plus grand pour pousser mes concitoyens à l'étude des lettres, que d'exposer le trésor encore caché de notre langue ?» M. Furno, « De la métaphore à la standardisation : l'intitulé des dictionnaires du latin et du français, 1500-1650 ", Le français préclassique, éd. citée, note 29.

41. Trad. M. Furno : «Dans cet ouvrage, l'usage de la langue française (bien que pas même cela semblât s'écarter grandement de notre projet), n'aurait certes pas relevé de notre dessein, si je n'avais pas dû obéir en tout à la volonté de certains hommes avec qui je ne pouvais être en désaccord, et qui me réclamaient ce point avec une grande insistance, d'autant qu'ils me demandaient quelque chose d'honnête, et qui serait peut-être même d'une certaine utilité à ceux qui, alors qu'ils veulent paraître bien instruits vis-à-vis du vulgaire, critiquent cela avec une grande liberté. Nous avons seulement ajouté les traductions qui se sont présentées d'évidence et sans périphrase, ne laissant pas de côté, de propos délibéré, (ces cas) peu nombreux. » M. Furno, «Les dictionnaires de Robert Estienne : sens et finalité d'une œuvre lexicographique », éd. citée, p. 25 , note 34 .

42. M. Furno, ibid., p. 25.

43. E. E. Brandon, op. cit., p. 1. J. Pruvost, op. cit., p. 25.

44. Nous citons le texte de G. Tory dans sa version de 1549 : L'art et science de la vraye proportion des Lettres Attiques (fin de l'épître non paginée «Aux Lecteurs...»). Robert Estienne connaît vraisemblablement Geoffroy Tory qui a travaillé comme correcteur pour Henri Estienne père.

45. Jacques Dubois (dit Sylvius), In linguam gallicam Isag医ge, unà cum ejusdem Grammatica latinogallica, ex Hebrceis, grcecis, \& Latinis authoribus, Paris, Robert Estienne, 1531. 
46. Ces explications sont données par R. Estienne dans la préface de sa grammaire : Robert Estienne, Traicté de la grammaire Francoise, Paris, Robert Estienne, 1557, « Au Lecteur », p. 3 : «Pourtant que plusieurs desirans avoir ample cognoissance de nostre langue Francoise, se sont plains à nous de ce qu'ils ne povoyent aiseement s'aider de la Grammaire Francoise de Maistre Lois Maigret (à cause des grans changemens qu'ils y voyoyent, fort contraires à ce qu'ils en avoyent jà apprins, principalement quant à la droicte escripture) ne de l'Introduction à la langue Francoise composée par M. Jaques Sylvius medecin (pourtant que souvent il a meslé des mots de Picardie dont il estoit)».

47. Ibid., p. 4.

48. Op. cit. p. 26.

49. B. Quemada, op. cit., p. 159 sq. : « Dictionnaires généraux et extensifs ».

50. " comme l'indique leur nom [...] les Thesaurus sont aussi des lieux patrimoniaux de la langue latine qui ont pour objet la conservation du plus grand nombre possible de mots, de formulce loquendi, et par là même d'exemples et d'extraits de textes, au point que certains auteurs semblent avoir été rigoureusement et complètement découpés en fragments pour constituer la matière du dictionnaire.» M. Furno, «Doctrina vel disciplina. Tensions et évolutions des dictionnaires latins humanistes, 1502-1636», éd. citée, p. 73.

51. Se reporter à la note précédente.

52. Les locutions désignées par la lettre B. sont le plus souvent des formules légales, empruntées à l'édition des Forensia de Budé, ouvrage inachevé qu'Estienne publie après la mort de l'auteur en 1544. Dans cet ouvrage Budé tentait de réformer le latin de la procédure en remplaçant les formules latines médiévales par des tournures classiques, de façon à créer « une langue juridique moderne et humaniste » (M. Furno, «Robert Estienne, imprimeur des Forensia », dans Qui écrit? Figures de l'auteur et des co-élaborateurs du texte $X^{e}$-XVIII siècle, dir. M. Furno, Paris, ENS Éditions, Institut d'histoire du livre, 2009, p. 191-205). Le classement de Budé étant thématique et non alphabétique, R. Estienne le double l'année suivante, en 1545, d'un volume d'index latin-français (avec une traduction par Du Luc des formules latines de Budé) et français-latin (ibid. p. 193 et 197). T. R. Wooldridge précise qu'elles proviennent des Adversaria de Budé, regroupées dans sept cahiers manuscrits qui constituent une partie des documents inédits que la famille de Budé a confié à Estienne après sa mort (Les Débuts de la lexicographie française. Estienne, Nicot et le Thresor de la langue françoyse (1606), Toronto, Le Net des Études françaises, 2010 [édition électronique en ligne], « Nomenclature qualitative », « Nomenclature annoncée », 2.1.1.1).

53. T. R. Wooldridge, ibid.

54. C. Beaulieux, «Les dictionnaires de la langue française du seizième siecle», Revue internationale de l'enseignement, mai-juin 1927, p. 155-167, spé. p. 157.

55. Au point que T.R. Wooldridge, considérant ce finale de la préface de 1549, déclare qu'Estienne envisage désormais «un dictionnaire du français, fait pour les Français, par les Français» et non plus un simple reflet du Dictionarium (op. cit., «Du dictionnaire latin au dictionnaire français » [du latin au français], 1.1.2.2).

56. J. Pruvost, se reporter supra n. 48.

57. Allusion au Thesaurus linguce latince publié par R. Estienne en 1531 et déjà réédité une première fois en 1536 (la troisième édition datant de 1543).

58. Le Dictionarium latino-gallicum, publié en 1538.

59. «tous les deux » désigne à la fois l'âge et l'état des connaissances. L'entreprise de R. Estienne concerne donc tout le monde, quel que soit son âge ou l'état de ses connaissances, avec un « ou » inclusif.

60. Allusion à l'édition princeps de 1539.

61. Le pronom sujet sous-entendu est «tu », désignant le lecteur. R. Estienne entend lui expliquer la présence de la lettre « $\mathrm{B} »$ qu'il ne pourrait comprendre sinon.

62. Se reporter supra note 52 . 
63. Nous remercions Madame Émilienne Molina, Conservatrice en Chef, section des fonds patrimoniaux, de ses informations concernant les exemplaires de 1539 et 1549 conservés dans cette bibliothèque. Madame Molina nous informe que, depuis 1983, les fonds de la bibliothèque du Musée Calvet ont été transférés dans la Livrée Ceccano (2 bis, rue Laboureur, Avignon).

64. Nous remercions Madame Claire Ben Kakhdar-Kreuwen, directrice-conservatrice en chef, pour nous avoir communiqué cette cote.

65. Nous remercions Madame Christine Le Bretton, directrice, qui nous signale qu'une mention manuscrite sur le catalogue ancien précise que l'ouvrage a désormais disparu.

\section{RÉSUMÉS}

Cet article s'insère dans le cadre d'une étude de l'engagement linguistique dans le paratexte des premiers dictionnaires des langues vernaculaires. Au sein du domaine français, la présentation des préfaces du Dictionaire Francoislatin de Robert Estienne (1539 et 1549) constitue un premier témoignage.

\section{INDEX}

Mots-clés : défense du français, langue vernaculaire, premier dictionnaire du français, préface de dictionnaire, Robert Estienne

Index géographique : domaine français

Index chronologique : XVIe siècle

\section{AUTEURS}

\section{SABINE LARDON}

Sabine Lardon est professeur de grammaire et rhétorique françaises des XVI ${ }^{\mathrm{e}}$ et XVII ${ }^{\mathrm{e}}$ siècles à l'Université Jean Moulin - Lyon 3. 